Review Article

\title{
NANOTECHNOLOGY : THE NEW ERA OF TECHNOLOGY
}

\author{
Laxman Singh Kaira ${ }^{1} \&$ Richa Singh ${ }^{2}$ \\ Senior Lecturer ${ }^{1}$, Intern ${ }^{2}$ \\ Department of Prosthodontics, Institute of Dental Sciences, Bareilly \\ Correspondence: \\ Laxman Singh Kaira
}

Room No. 97, New Resident Hostel, Institute of Dental Sciences, Bareilly Mobile : NU - +9195289 31095: E-mail : luckysinghkaira111@gmail.com

\begin{abstract}
:
Nanotechnology is a natural end result of scientific development and our ability to understand and manipulate matter at smaller and smaller level. Current research is directed towards the production of wide array of different nano-scale structures. The fabrication techniques of the structures can be divided into 2 approaches: "top-down" and "bottom-up." New potential treatment opportunities in dentistry may include, local anesthesia, dentition re-naturalization, and permanent hypersensitivity cure, complete orthodontic realignments during single office visit, covalently bonded diamondised enamel, and continuous oral health maintenance using mechanical dentifrobots. Nanotechnology is foreseen to change health care in a fundamental way by providing novel methods for disease diagnosis and prevention, therapeutics selection, tailored to the patients profile, drugdelivery and gene therapy.
\end{abstract}

Keywords: Nanodentistry, Technology, dentifrobots.

\section{Introduction:}

Nano is derived from Greek word for "dwarf". Nanotechnology is the science of manipulating matter measured in the billionths of meters or nanometer, roughly the size of 2 or 3 atoms (1).

Nanoscience does not describe practical application but rather the scientific study of the properties of nanomeric world.

Nanotechnology is a natural end result of scientific development and our ability to understand and manipulate matter at smaller and smaller levels. In 1959 the renowned physicist Richard P. Feynman speculated the potential of nanosize devices in his historic lecture "There's plenty of room at the bottom." The vision of nanotechnology was born. The term 'nanotechnology' was coined by Prof. Kerie

E. Drexeler, in 1980's who

Access this article online
Quick Response Code
promoted the technological significance of nanoscale phenomena (2). The basic idea of nanotechnology, used in narrow sense of the word, is to employ individual atoms and molecules to construct functional structures $(3,4)$.

There are two perspectives on how nano products are made

1) Building up particles by combining atomic elements.

2) Using equipments to create mechanical nanoscale objects.

Current research is directed towards the production of wide array of different nanoscale structures. The fabrication techniques of the structures can be divided into 2 approaches: "top-down" and "bottom-up" (4).

\section{THETECHNIQUES (5)}

\section{Top-down technique}

These seek to create smaller devices by using larger ones to direct their assembly. _ Bottom-up technique: These seek to arrange smaller components into more complex assembly. The various nanostructures that are in use at present are Nanopores, Nanotubes, Quantum dots, Nanoshells and Dendrimer (6). Nanodentistry: is the science and technology of maintaining near-perfect oral health through the use of nanomaterials including tissue engineering and nanorobotics (3)

\section{NANODENTISTRY}

New potential treatment opportunities in dentistry may 
include, local anesthesia, dentition re-naturalization, and permanent hypersensitivity cure, complete orthodontic realignments during single office visit, covalently bonded diamondised enamel, and continuous oral health maintenance using mechanical denti-frobots. When the first micro-size dental nano-robots can be constructed, dental nanorobots might use specific motility mechanisms to crawl or swim through human tissue with navigational precision, acquire energy, sense, and manipulate their surroundings, achieve safe cytopenetration and use any of the multitude techniques to monitor,

interrupt, or alter nerve impulse traffic in individual nerve cells in real time. These nanorobot functions may be controlled by an onboard nanocomputer that executes preprogrammed instructions in response to local sensor stimuli. Alternatively, the dentist may issue strategic instructions by transmitting orders directly to in vivo nanorobots via acoustic signals or other means (7).

\section{Local anesthesia}

In the era of nanotechnology, a colloidal suspension containing millions of active analgesic micron-size dental robots will be instilled on the patient's gingiva. After contacting the surface of crown or mucosa, the ambulating nanorobots reach the pulp via the gingival sulcus, lamina propria and dentinal tubules guided by combination of chemical gradients, temperature differentials and even positional navigation all under the control of on board nanocomputer as directed by the dentist $(3,7)$. Once installed in the pulp, the analgesic dental robots may be commanded by the dentist to shut down all sensitivity in any particular tooth that requires treatment. After oral procedures are completed, the dentist orders the nanorobots to restore all sensation, to relinquish control of nerve traffic and to egress from the tooth by similar pathwaysused for ingress (7).

\section{Tooth Repair}

Nanodental techniques for major tooth repair may evolve through several stages of technological development, first using genetic engineering, tissue engineering and tissue regeneration and later growing whole new teeth invitro and installing them. Ultimately, the nanorobotic manufacture and installation of a biologically autologous whole replacement tooth including both mineral and cellular components e.g., complete dentition replacement therapy, should become feasible (7).

\section{Tooth Renaturalization}

Dentition renaturalization procedures may become a popular addition to the typical dental practice, providing perfect methods for esthetic dentistry. This trend may begin with patients who desire to have their old dental amalgams excavated and their teeth remanufactured with native biological materials. But demand will grow for full coronal renaturalizations in which all fillings, crowns, and other necessary $20^{\text {th }}$ century modifications to the visible dentition are removed, with the affected teeth remanufactured so as to be indistinguishable from the natural originals(7).

\section{Dentin Hypersensitivity}

Natural hypersensitive teeth have eight times higher surface density of dentinal tubules and diameter with twice as large as nonsensitive teeth. Reconstructive dental nanorobots, using native biological materials, could selectively and precisely occlude specific tubules within minutes, offering patients' quick and permanent cure (7).

\section{Dentifrobots}

Subocclusal dwelling nanorobotic delivered by mouthwash or toothpaste could patrol all supragingival and subgingival surfaces at least once a day, metabolizing trapped organic matter into harmless and odorless vapors and performing continuous calculus debridement. These invisibly small dentifrobots [1-10 micron], crawling at 1- 10 microns/sec, would be inexpensive, purely mechanical devices, that would safely de-activate themselves if swallowed and would be programmed with strict occlusal avoidance protocol (8).

\section{Nanoneedles}

Suture needles incorporating nano-sized stainless steel crystals have been developed. Nanotweezers are also under development which will make cell-surgery possible in the near future 8). 


\section{Dental durability and cosmetics}

Tooth durability and appearance may be improved by replacing upper enamel layers with pure sapphire and diamond which can be made more fracture resistant as nanostructured composites, possibly including embedded carbon nanotubes (7).

\section{Orthodontic treatment}

Orthodontic nanorobots could directly manipulate the periodontal tissues, allowing rapid and painless tooth straightening, rotating and vertical repositioning within minutesto hours (7).

\section{Photosensitizersand carriers}

Quantum dots can be used as photosensitizers and carriers. They can bind to the antibody present on the surface of the target cell and when stimulated by UV light, they can give rise to reactive oxygen species and thus will be lethal to the target cell (7).

Diagnosis of oral cancer and other diseases - Nano Electromechanical Systems (NEMS) Nanotechnology based NEMS biosensors that exhibit exquisite sensitivity and specificity for analyte detection, down to single molecule level are being developed. They convert (bio) chemical to electrical signal (9).

\section{Oral Fluid NanoSensor Test (OFNASET)}

The Oral Fluid NanoSensor Test (OFNASET) technology is used for multiplex detection of salivary biomarkers for oral cancer. It has been demonstrated that the combination of two salivary proteomic biomarkers (thioredoxin and IL-8) and four salivary mRNA biomarkers (SAT, ODZ, IL-8, and IL1b) can detect oral cancer with high specificity and sensitivity (10).

\section{Optical Nanobiosensor}

The nanobiosensor is a unique fiberoptics-based tool which allows the minimally invasive analysis of intracellular components such as cytochrome c, which is a very important protein to the process which produces cellular energy and is well-known as the protein involved in apoptosis, or programmed cell death (11).

\section{Treatment of oral cancer(7)}

Nanomaterials For Brachytherapy BrachySilTM (Sivida, Australia) delivers 32P, clinical trial.

\section{Drug Delivery Across The Blood-Brain Barrier}

More effective treatment of brain tumours, Alzheimer's, Parkinson's in development

\section{Nanovectors For Gene Therapy}

Non-viral gene delivery systems

\section{Photodynamic Therapy-}

Hydrophobic porphyrins are potentially interesting molecules for the photodynamic therapy (PDT) of solid cancers or ocular vascularization diseases (12).

\section{Nanocomposites}

Nanoproducts Corporation has successfully manufactured nonagglomerated discrete nanoparticles that are homogeneously distributed in resins or coatings to produce nanocomposites. The nanofiller used include an aluminosilicate powder having a mean particle size of 80 $\mathrm{nm}$ and a 1:4 $\mathrm{M}$ ratio of alumina to silica and a refractive index of 1.508(13).

Trade name: Filtek ${ }^{\mathrm{tm}}$ Supreme universal restorative pure Nano ${ }^{\text {tm }}$

\section{Advantages of Nanofillers in dental composites $(14,15,16)$ :}

1. Properties are similar to liquid - do not thicken the resin .

2. Size below absorption of visible light $(0.4-0.8 \mathrm{~mm})$ invisible.

3. Enhance the polish-ability of resin .

4. Extreme surface to volume ratio and the ability to fit between several polymer chains - high filler loading in workable consistencies.

5. Increase hardness and wear resistance

6. $50 \%$ reduction in polymerization shrinkage and less staining.

7. Superior hardness, translucency and esthetic appeal.

8. Superior flexural strength

9. Superior modulus of elasticity 


\section{Nanosolution (14)}

Nanosolutions produce unique and dispersible nanoparticles, which can be used in bonding agents. Types of nanoparticles

1 Nanomeric: These are monodisperse non aggregated and non agglomerated silica nanoparticles. They reduce the interstitial spacing and increase the filler loading.

2 Nanoclusters: These are zirconia-silica particles (2 to 20 $\mathrm{nm}$ ) and zirconyl salt (from $75 \mathrm{~nm}$ ) which are spheroidal agglomerated particles. They have Dentin, Enamel and body shades because of radiopacity and there is high gloss retention with silica nanomer.

Adper $^{\text {tm }}$ single bond 2 adhesives incorporates $10 \%$ by weight of $5 \mathrm{~nm}$ diameter spherical silica particles through a process that prevents agglomeration. As discrete particles,their extremely small size keep them in colloidal suspension.

Trade name- Adper $^{\text {tm }}$ single bond plus adhesives single bond 2 .

\section{Nano-optimised mouldable ceramics}

Nanofillers: Enhance polish ability and reduce wear.

Nanopigments: Adjust the shade of the restoration to the surrounding teeth (chameleon effect).

Nanomodifiers: Increase the stability (non-slump) of the material and prevent sticking to instruments (17).

\section{Impression Materials}

Nanofillers are integrated in vinylpolysiloxanes, producing a unique addition of siloxane impression materials. The material has better flow, improved hydrophilic properties and enhanced detail precision (13).

Trade name- Nanotech elite H-D.

\section{Nanoencapsulation}

SWRI [South West Research Institute] has developed targeted release systems that encompass nanocapsules including novel vaccines, antibiotics and drug delivery with reduced side effects.

At present, targeted delivery of genes and drugs to human liver has been developed by Osaka University, Japan in 2003. Engineered Hepatitis B virus envelope $L$ particles were allowed to form hollow nanoparticles displaying a peptide that is indispensable for liver-specific entry by the virus in humans. Future specialized nanoparticles could be engineered to target oral tissues, including cells derived from the periodontium (7).

\section{Discussion :}

There is growing body of scientific evidence which demonstrates the potential for some nanomaterials to be toxic to humans or the environment. Studies have demonstrated the potential for nanomaterials to cause DNA mutation and induce major structural damage to mitochondria and affect the regulatory mechanisms of enzymes and other proteins, even resulting in cell death (3, 18).

Nanotechnology is facing certain challenges like precise positioning and assembly at molecular scale part, economical nanorobots mass production technique and simultaneous coordination of activities of large number of independent nanorobots, biocompatibility and social issues of public acceptance, ethics, regulation and human safety (7).

Nanotechnology is foreseen to change health care in a fundamental way by providing novel methods for disease diagnosis and prevention, therapeutics selection, tailored to the patients profile, drug delivery and gene therapy.

\section{Summary:}

'The emerging fields of nanoscale science, engineering and technology, the ability to work at molecular level, atom by atom, to create large structures with fundamentally new properties and functions are leading to unprecedented understanding and control over the basic building blocks and properties of natural and man-made things (13). As with all emerging technologies, a successful future for nanotechnology will only be achieved through open sharing of ideas and research findings. 


\section{References:}

1. Kaehler T. Nanotechnology, Basic concepts and Definitions. Clinical Chem 1994;40(9):1797-99.

2. Zones AZ. Nanotechnology - Introduction to Nanotechnology. Physics. about.Com/od/nanotechnology.

3. Freitas RA J r. Nanodentistry. J Am Dent Assoc 2000;131(11):1559-63.

4. Handbook of Nanoscience and Nanotechnology.htm.en.wikipedia. org / wiki/The opensource-Handbook of Nanoscience and Nanotechnology.

5. Silva GA. Introduction to nanotechnology and its applications to medicine. Surg Neurol 2004;61:216-20.

6. Janes DB. What is Nanotechnology? Purdue University, The Janes Group Webpage, 2002. cobweb.ecn.purdue. edu/janes/ prof_david_janes

7. Saravana RK, Vijayalaksmi R. Nanotechnology in dentistry. Ind J Dent Res 2006;17(2):62-65.

8. Patil M, M ehta DS. Future impact of Nanotechnology on M edicine and Dentistry. J Indian Soc Periodontol 2008; 12(2):34-40.

9. Li Y, Denny P, Ho CM. The Oral Fluid MEM S/NEMS Chip (OFM NC): Diagnostic and Translational Applications. Adv Dent Res 2005;18:3-5.

10. Gau V, Wong D. Oral fluid nanosensor test (OFNASET) with advanced electrochemical-based molecular analysis platform. Ann NY Acad Sci 2007;1098:401-10.
11. Song JM , Kasili PM , Griffin GD, Vo-Dinh T. Detection of cytochrome Cin a single cell using an optical nanobiosensor. Anal Chem 2004;76(9):2591-2594

12. Vargas A, Pegaz B, Debefve E. Improved photodynamic activity of porphyrin loaded into nanoparticles: An in vivo evaluation using chick embryos. Int J Pharm 2004; 286(1-2):131-45.

13. Jhaveri HM, Balaji PR. Nanotechnology, The future of dentistry. J Indian Prosthodont Soc 2005;5:15-17.

14. Mitra SB, Wu D, Holmes BN. An application of nanotechnology in advanced dental materials. J Am Dent Assoc 2003;134(10):1382-90.

15. Kim JS, Cho BH, Lee IB. Effect of the hydrophilic nanofiller loading on the mechanical properties and the microtensile bond strength of an ethanolbased one-bottle dentin adhesive. J Biomed M ater Res B Appl Biomater 2005;72(2):284-91.

16. Senawongse $P$, Pongprueksa $P$. Surface roughness of nanofill and nanohybrid resin composites after polishing and brushing. J Esthet Restor Dent 2007;19(5):265-73.

17. Schirrmeister JF, Huber K, Hellwig E, Hahn P. Two-year evaluation of a new nanoceramic restorative material. Clin Oral Investig 2006;10(3):181-86

18. Freitas RA J r. Nanotechnology, nanomedicine and nanos 